\section{A simple technique to avoid suturing in the drain during total knee arthroplasty}

\section{Williams, K Sri-Ram}

Royal National Orthopaedic Hospital NHS Trust, UK

\section{CORRESPONDENCE TO}

Daniel Williams, E: williams-dan@doctors.org.uk

\section{BACKGROUND}

Closure of the arthrotomy following total knee arthroplasty is not without risk. Most orthopaedic surgeons are aware of cases when a drain has been caught by a suture during closure so that it cannot be removed, resulting in return to theatre, an intra-articular foreign body or infection.

\section{TECHNIQUE}

Before closing the arthrotomy, pass the drain through the soft tissues lateral to the proximal end of the wound, leaving the distal end long

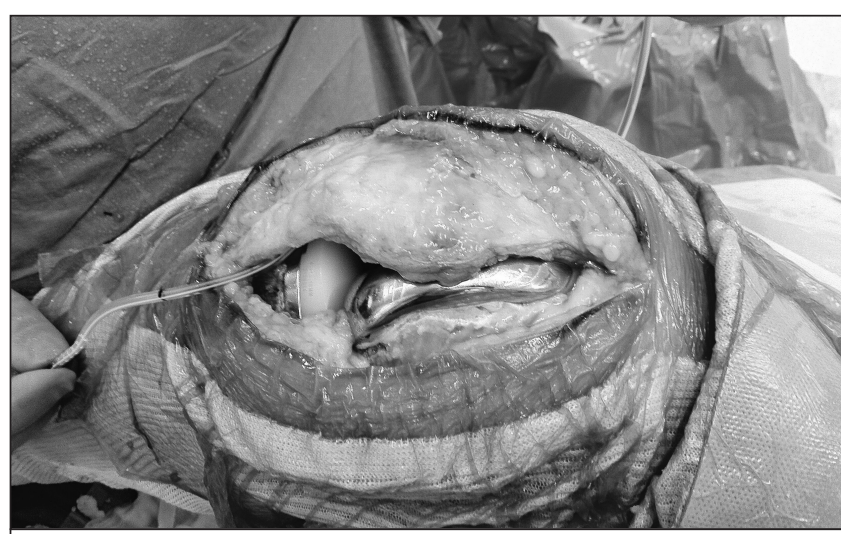

Figure 1 Clinical photograph showing the drain passing through the soft tissues lateral to the proximal end of the wound, leaving the distal end long

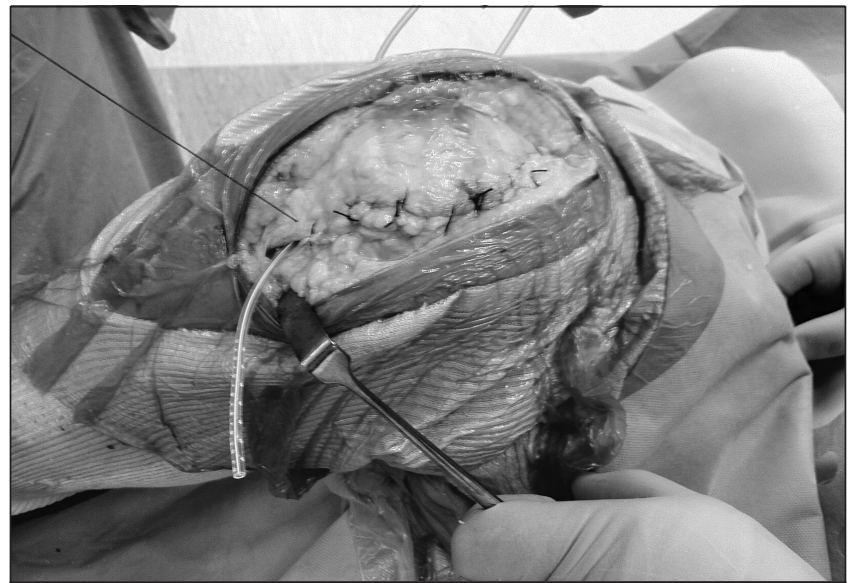

Figure 2 Clinical photograph demonstrating the long distal drain still protruding after closure of the arthrotomy
(Fig 1). Close the arthrotomy from proximal to distal, leaving the distal end protruding (Fig 2). As you reach the end of the wound where the drain exits the arthrotomy, withdraw the drain to length. As the drain withdraws easily, you can be sure that it has not been sutured into the wound. If it cannot be removed at this stage, the arthrotomy is easily explored.

\section{DISCUSSION}

This simple technique reduces the chances and problems associated with a drain that cannot be removed after total knee arthroplasty. Although we acknowledge that this technique may not be novel, we would like to draw attention to its usefulness and feel it can be widely used in other procedures that have difficult closures.

\section{Use of Johan forceps as endoloop pushers for laparoscopic appendicectomy}

\section{K Siddique ${ }^{1}$, N Siddiqi ${ }^{1}$, P Sedman ${ }^{2}$}

${ }^{1}$ Wirral University Teaching Hospital NHS Foundation Trust, UK

${ }^{2} \mathrm{Hull}$ and East Yorkshire Hospitals NHS Trust, UK

\section{CORRESPONDENCE TO}

Khurram Siddique, E: sk.sid@hotmail.co.uk

\section{BACKGROUND}

Laparoscopic appendicectomy is one of the most common surgical procedures and is often performed out of hours. Securing the base of the appendix may be achieved either by stapling or by use of a preformed endoloop. ${ }^{1}$ Stapling is an expensive option and mandates the use of a $12 \mathrm{~mm}$ trocar. Pre-formed single-use endoloops with singleuse (often integral) knot pushers may not always be accessible. We present an inexpensive alternative to the pre-formed endoloop and a technique to safely push the knot using universally available $5 \mathrm{~mm}$ laparoscopic forceps.

\section{TECHNQIUE}

An endoloop is created extracorporeally using a Roeder knot in a standard fashion. ${ }^{2}$ The standing end is kept long and passed through

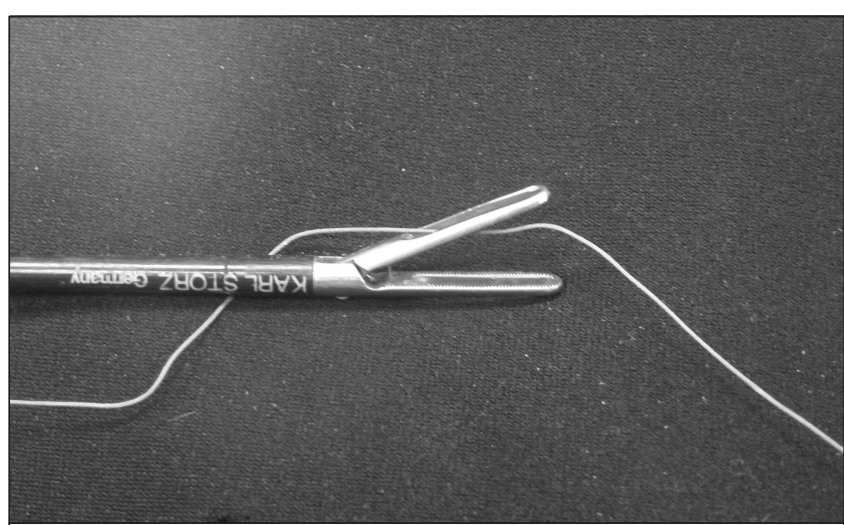

Figure 1 Long end of endoloop passed through the upper jaw of Johan forceps 
the fenestration of the mobile jaw of a Johan forceps (Fig 1). The jaws of the forceps are then loosely approximated behind the knot to act as the knot pusher (Fig 2) and this ensures smooth delivery of

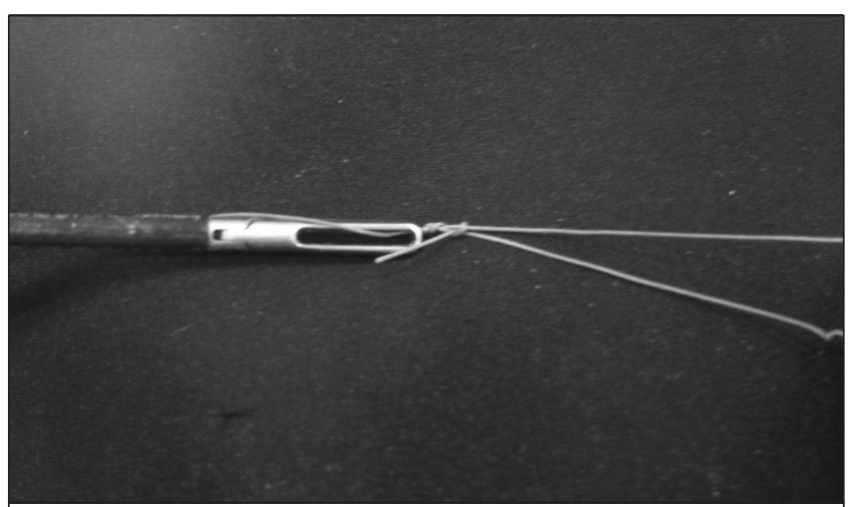

Figure 2 Tip of Johan forceps in close approximation with the knot

the ligature (Fig 3). Care is needed to ensure the jaws are not closed too tightly as the serrations may shred the suture material. We have found the optimal suture to be a size 1 Vicry ${ }^{\circledR}$ suture (Ethicon Inc, Somerville, NJ, US), which balances knot security with thread robustness and strength. Monofilaments are an alternative.

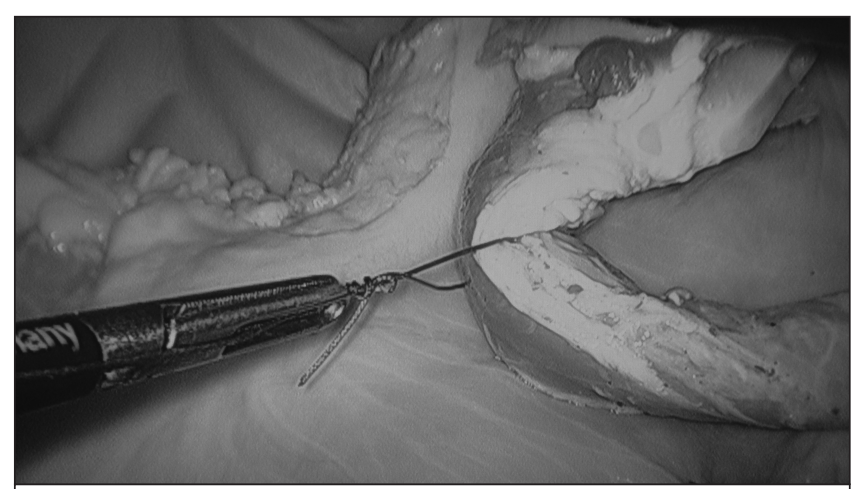

Figure 3 Johan forceps tip acting as a guide to help controlled placement of knot

The advantage of passing the standing end through the fenestration as shown is that the knot is always easily retrieved should the forceps and standing end become misaligned during the pushing process. The technique is equally applicable to loops created extracorporeally and used in a lasso fashion for pedicle ligation (Fig 4) and for sutures passed intracorporeally but tied extracorporeally. In the latter case, greater care is required to define the standing end during the knot tying process.

\section{DISCUSSION}

The self-created endoloops are reliable, safe and cost effective. ${ }^{3}$ The base of the appendix can be secured safely without the need for expensive commercial endoloops or a knot pusher, thereby significantly reducing costs. This technique is also adaptable for the creation of extracorporeal knots for ligation in continuity as might be desired for

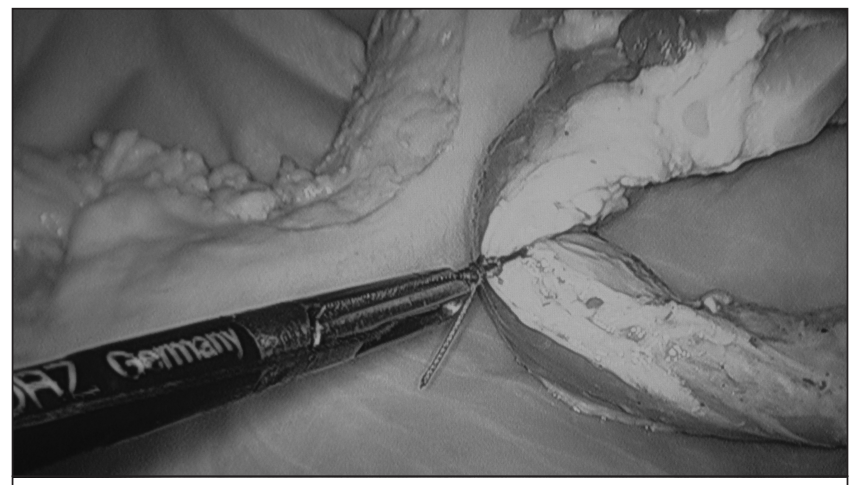

Figure 4 Secure and precise placement of knot at the desired site for ligature tightening

tying off an appendicular artery. Our technique has the added benefits of secure knot placement at the correct anatomical site with the help of a Johan forceps, which has not been described previously.

\section{References}

1. Lukish J, Powell D, Morrow S et al. Laparoscopic appendectomy in children: use of the endoloop vs the endostapler. Arch Surg 2007; 142: 58-61.

2. Steele RJ. Roeder knot for tight corners in conventional abdominal surgery. $J R$ Coll Surg Edinb 1991; 36: 412

3. Yildiz F, Terzi A, Coban $\mathrm{S}$ et al. The handmade endoloop technique. A simple and cheap technique for laparoscopic appendectomy. Saudi Med J 2009; 30 224-227.

\section{A simple way to allow continuous ventilation during tracheostomy}

G Chawdhary, P Silva, A Lamyman

Oxford University Hospitals NHS Trust, UK

\section{CORRESPONDENCE TO}

Gaurav Chawdhary, E: gauravchawdhary@gmail.com

During open tracheostomy, formation of the tracheal window may puncture the endotracheal tube cuff inadvertently. This causes loss of

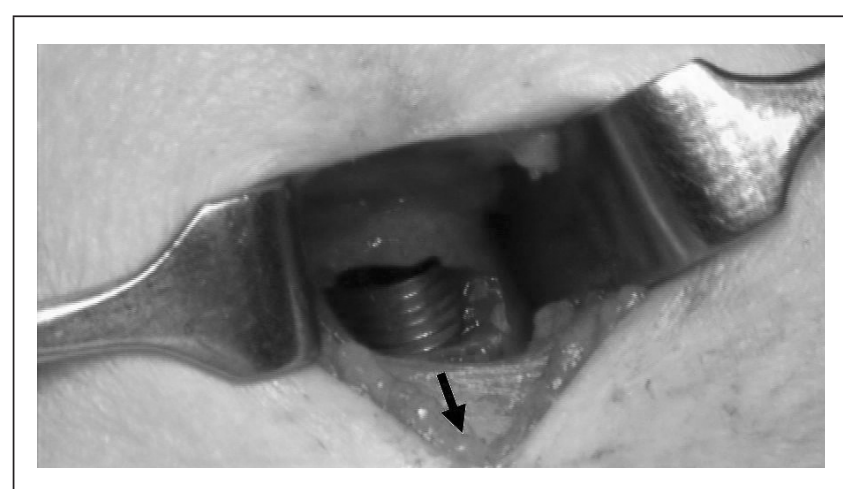

Figure 1 Operative photograph showing tracheostomy: The endotracheal tube has been advanced caudally to ensure the cuff is safe when forming the tracheal window. 